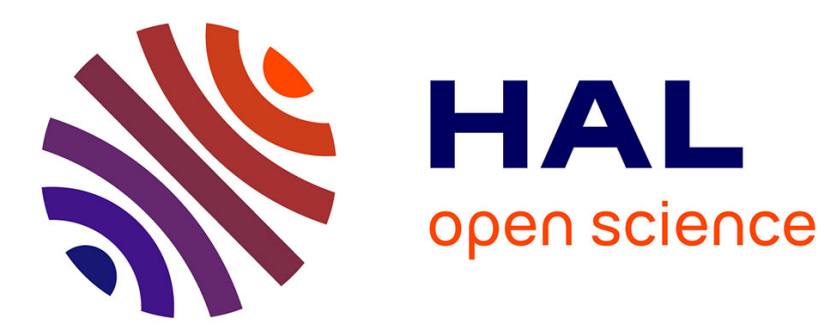

\title{
Etude XPS de surfaces de nylon 6 traitées par un plasma d'oxygène en post-décharge
}

\author{
A. Scheuer, J. Hommet, J.-P. Deville
}

\section{To cite this version:}

A. Scheuer, J. Hommet, J.-P. Deville. Etude XPS de surfaces de nylon 6 traitées par un plasma d'oxygène en post-décharge. Journal de Physique IV Proceedings, 1996, 06 (C4), pp.C4-451-C4-459. 10.1051/jp4:1996441 . jpa-00254324

\section{HAL Id: jpa-00254324 https://hal.science/jpa-00254324}

Submitted on 1 Jan 1996

HAL is a multi-disciplinary open access archive for the deposit and dissemination of scientific research documents, whether they are published or not. The documents may come from teaching and research institutions in France or abroad, or from public or private research centers.
L'archive ouverte pluridisciplinaire HAL, est destinée au dépôt et à la diffusion de documents scientifiques de niveau recherche, publiés ou non, émanant des établissements d'enseignement et de recherche français ou étrangers, des laboratoires publics ou privés. 


\title{
Etude XPS de surfaces de nylon 6 traitées par un plasma d'oxygène en post-décharge
}

\author{
A. Scheuer, J. Hommet et J.-P. Deville \\ Institut de Physique et Chimie des Matériaux de Strasbourg, UMR 0046 du CNRS, 23 rue du Loess, \\ BP. 20 CR, 67037 Strasbourg, France
}

\begin{abstract}
Résumé : L'étude in situ par XPS des surfaces de Nylon 6 traitées par un plasma d'oxygène pur en post-décharge montre que les modifications des liaisons chimiques dues au traitement dépendent fortement des doses d'oxygène atomique $\left(\mathrm{at} / \mathrm{cm}^{2} / \mathrm{s}\right)$ atteignant la surface. Les faibles doses limitent l'incorporation d'oxygène à la surface alors que les traitements réalisés aux fortes doses induisent des coupures et des suppressions de chaines aliphatiques qui ont pour effet d'augmenter le taux d'oxygène à la surface. La première réaction qui se produit lors du traitement est la coupure de la liaison faible C-N. Les études de remises à l'air entre le traitement et l'analyse montrent que les réactions qui se produisent dépendent des modifications de la surface. Une surface traitée dans des conditions assez peu dégradantes reste stable lors d'une longue exposition à l'air.
\end{abstract}

\begin{abstract}
In situ XPS studies of Nylon 6 surfaces treated in the post-discharge of an oxygen plasma show the link between the atomic oxygen doses $\left(\mathrm{at} / \mathrm{cm}^{2} / \mathrm{s}\right.$ ) and the modification of the surfaces. Incorporation of oxygen at the surface is limited for small doses whereas large doses induce a loss of aliphatic fragments which causes an increase of the oxygen bonds grafted at the surface. The first reaction occuring at the surface is the breaking of the weak $\mathrm{C}-\mathrm{N}$ bond.A weakly degraded surface remains stable even after a long exposure in air.
\end{abstract}

\section{INTRODUCTION}

Le traitement de surface des polymères par plasma s'est considérablement développé ces dernières années. En effet, il permet de modifier les surfaces des polymères sans pour autant affecter leurs propriétés de volume. Il existe de nombreux types de réacteurs ayant leurs propres conditions de décharge (mode d'excitation, fréquence de la décharge, puissance fournie, pression du milieu réactionnel, gaz utilisés, ...). Les traitements diffèrent aussi suivant qu'ils ont lieu dans la décharge même ou en post-décharge, milieu où seules sont présentes les espèces à temps de vie assez long et où ne subsiste plus aucune espèce chargée. L'avantage de réaliser des traitements en post-décharge est considérable sur deux points. D'une part, l'absence d'espèces chargées ou d'autres espèces à taux de réactivité importants évite ou diminue les phénomènes de dégradation de la surface à traiter et permet donc un traitement moins destructif qui se révèle, en général, plus stable dans le temps. D'autre part, le nombre réduit d'espèces présentes permet de modéliser le milieu réactionnel et aussi de relier les modifications de surface aux densités des espèces qui entrent en jeu dans le processus de traitement.

Le choix des gaz de traitement dépend des propriétés désirées pour la surface traitée. Une surface pourra être rendue hydrophobe par des traitements aux gaz fluorés [1] ou au contraire hydrophyle par emploi de gaz oxygénés [2]. L'utilisation de mélanges oxygène/azote peut 
avoir pour but d'augmenter la densité d'oxygène atomique en post-décharge sans modifier les autres paramètres de la décharge [3].

L'utilisation de la spectroscopie de photoélectrons induits par les rayons X (XPS) est entièrement justifiée dans le cadre de cette étude. En effet, pour caractériser les modifications de la surface tant au niveau quantitatif (enrichissement en oxygène) que qualitatif (type des fonctions créées), l'XPS est un outil très bien adapté. Le faisceau de photons X est reconnu pour peu détruire les structures chimiques des échantillons fragiles, comme le sont notamment les polymères, contrairement aux faisceaux d'électrons qui induisent des coupures de chaînes et donc des dégradations de surface. Il apporte par ailleurs des informations quantitatives à travers les rapports des aires de pics et qualitatives par la décomposition de spectres $\mathrm{C} 1 \mathrm{~s}$ et O1s, par exemple. L'originalité de cette étude réside aussi dans le montage de l'XPS utilisé qui permet d'analyser in situ les échantillons traités sans être obligé de faire une remise à l'air. On obtient ainsi des informations directes sur les effets du traitement. Cependant, pour avoir une information complète sur l'état chimique et structural des surfaces traitées, des analyses complémentaires ex situ sont nécessaires. La remise à l'air entre le traitement et l'analyse XPS est aussi requise pour les comparaisons et les études de réactions de post-oxydation (vieillissement).

L'étude décrite ici porte sur le traitement de surfaces de Nylon 6 (N6) en post-décharge de plasmas microondes $(2,45 \mathrm{Ghz})$ d'oxygène. Des analyses précédentes sur le traitement du polyéthylène et de la polycaprolactone [4] ont montré que les phénomènes de fonctionnalisation et de dégradation sont très complexes et dépendent notamment de la structure chimique et conformationnelle du polymère. C'est pourquoi les études du Nylon 6 du fait de la présence de la liaison faible $\mathrm{C}-\mathrm{N}$ présentent un intérêt certain dans la compréhension des mécanismes de traitement.

\section{PARTIE EXPERIMENTALE}

Le dispositif expérimental utilisé consiste en un réacteur de traitement plasma couplé à une enceinte d'analyse XPS suivant le schéma de la figure 1.

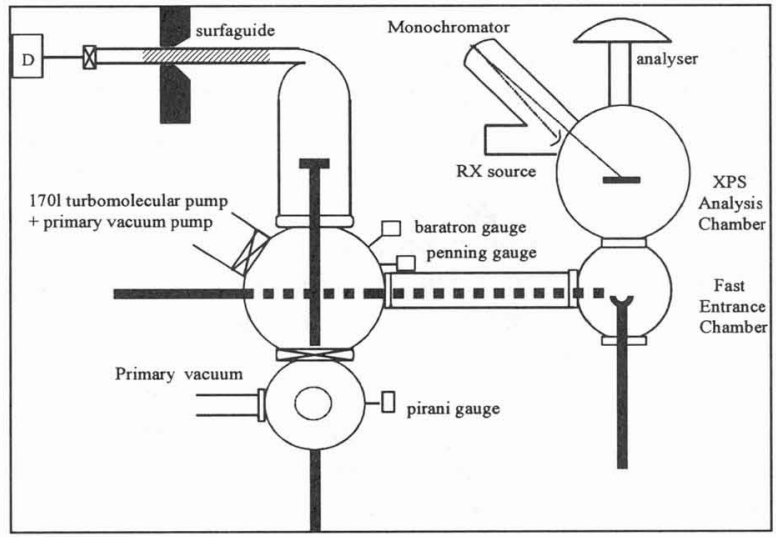

Figure 1 : Schéma du couplage réacteur de traitement - XPS.

Experimental setup : coupling between the treatment reactor and the XPS apparatus. 


\subsection{Dispositif de traitement plasma. Paramètres de traitement}

Le plasma est créé dans un réacteur en quartz par un générateur microonde $(2,45 \mathrm{GHz})$ SAIREM GMP $12 \mathrm{KE}(0-1200 \mathrm{~W})$ via un surfaguide. Le réacteur est constitué de deux tubes de 16 et $100 \mathrm{~mm}$ de diamètre. Ces deux tubes sont reliés entre eux par l'intermédiaire d'un coude à $90^{\circ}$. Les traitements ont lieu en post-décharge dans le tube de grand diamètre à une distance $X_{2}$ que l'on peut faire varier. La position du surfaguide est, elle aussi, variable, ce qui permet de maintenir une distance $\mathrm{X}_{1}$, constante et égale à $13 \mathrm{~cm}$, entre la fin de la décharge et le bout du tube de petit diamètre (fig. 2).

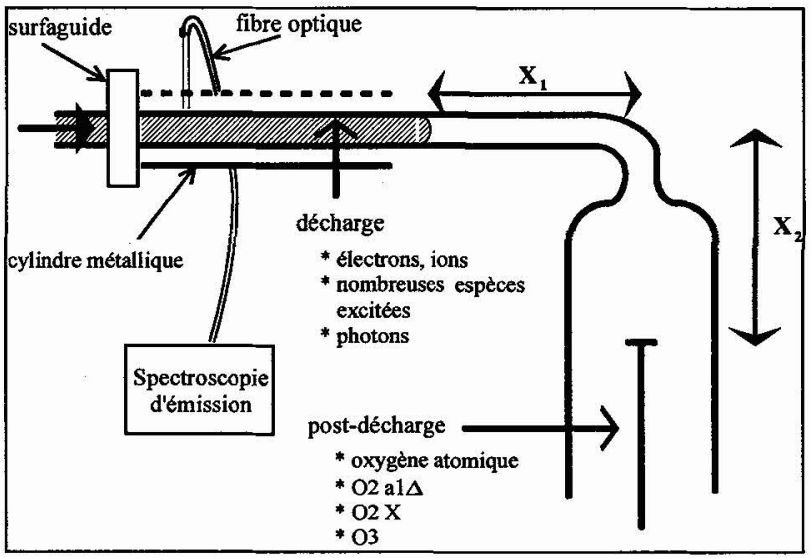

Figure 2 : Schéma du réacteur plasma.

Reactor schematics.

La présence du coude empêche les photons produits dans la décharge d'atteindre l'échantillon à traiter et évite des réactions de photooxydation.

$\mathrm{O}_{2}$ et $\mathrm{N}_{2}$ sont respectivement fournis par Prodair et Air Liquide ( $99 \%$ de pureté) et sont introduits directement dans la partie amont du tube de petit diamètre. Le flux des gaz est contrôlé par des régulateurs massiques d'Air Liquide Alphagaz.

Un système de pompage primaire permet d'obtenir la pression désirée pour le traitement et un système de pompage secondaire $\left(10^{-8}\right.$ mbar) évite la contamination des parois du tube en quartz entre les traitements.

Les paramètres de la décharge sont réunis dans le tableau I :

Tableau I : Paramètres de la décharge utilisés dans les traitements du Nylon 6 Discharge parameters used in Nylon 6 treatments

\begin{tabular}{|c|c|}
\hline & Nylon 6 \\
\hline puissance (W) & 300 \\
\hline pression (mbar) & 2,67 \\
\hline flux total (sccm*) & 20 \\
\hline gaz utilisés & $\mathrm{O}_{2}$ \\
\hline
\end{tabular}

* sccm : standard cubic centimeter per minute : $\mathrm{cm}^{3} \cdot \mathrm{min}^{-1}$ 


\subsection{Analyse XPS}

Le dispositif XPS couplé au réacteur de traitement plasma est un spectromètre VSW opérant avec une source AlK $\alpha$ monochromatisée. L'analyseur est hémisphérique de type HA150. Les électrons sont récoltés sur un multidétecteur. La pression qui règne dans l'enceinte d'analyse est de l'ordre de $6.10^{-10}$ à $1.10^{-9}$ mbar.

On travaille en mode énergie passante fixée (FAT). L'énergie passante est fixée à $90 \mathrm{eV}$ pour l'acquisition de spectres étendus et à $25 \mathrm{eV}$ pour les spectres haute résolution (pics $\mathrm{C} 1 \mathrm{~s}, \mathrm{O} 1 \mathrm{~s}$, $\mathrm{N} 1 \mathrm{~s}$, ...). La compensation de charge se fait à l'aide d'un canon à électrons de très faible énergie cinétique $(1 \mathrm{eV})$. Le spectre en énergie est obtenu avec une résolution de $0,45 \mathrm{eV}$ pour un FAT de $25 \mathrm{eV}$. La largeur à mi-hauteur du pic C1s d'un polyéthylène non traité est de 1,10 $\mathrm{eV}$ et celle d'un pic C1s de diamant est de $0,97 \mathrm{eV}$ sous les mêmes conditions d'analyse et de compensation de charge (FAT : $25 \mathrm{eV}$, énergie des électrons de compensation : $1 \mathrm{eV}$ ).

Les études d'évolution des spectres sous les faisceaux de rayons $\mathrm{X}$ et d'électrons montrent que les échantillons de Nylon 6 non traités sont assez stables pendant une heure d'analyse mais que les surfaces traitées lorsqu'elles présentent des liaisons très oxydées sont beaucoup plus fragiles et instables même pour des temps d'expositions très courts (environ $10 \mathrm{~min}$ ). Ainsi, les contributions des atomes de carbone fortement liés aux atomes d'oxygène pourront être un peu sous-estimées.

Dans l'analyse des spectres, tous les pics $\mathrm{C} 1 \mathrm{~s}$ sont référencés à $285,0 \mathrm{eV}$. Les aires des pics sont déterminées en soustrayant le bruit de font par la méthode de Shirley et corrigées par les coefficients de Scofield [5] pour obtenir les rapports O/C, N/O et N/C. Pour décomposer les pics $\mathrm{C} 1 \mathrm{~s}$ et $\mathrm{O} 1 \mathrm{~s}$ nous utilisons un programme réalisé au laboratoire [6] qui s'appuie sur les travaux théoriques de Doniach et Sunjic [7]. L'écart en énergie entre les différentes composantes a été déterminé dans plusieurs travaux [8] et les largeurs à mi-hauteur des lorentziennes (FWHM) ont été gardées constantes entre les spectres d'un même pic obtenu après différents traitements. Les notations utilisées pour les composantes des pics $\mathrm{C} 1 \mathrm{~s}$ sont représentées dans le tableau II :

Tableau II : Composantes des pics Cls

Components of the Cls peaks

\begin{tabular}{|c|c|c|c|c|c|c|}
\hline & $\mathrm{C} 1$ & $\mathrm{C} 2$ & $\mathrm{C} 3$ & $\mathrm{C} 4$ & $\mathrm{C} 5$ & $\mathrm{C} 6$ \\
\hline $\begin{array}{c}\text { position du } \\
\text { pic (eV) }\end{array}$ & 285,00 & 285,70 & $\begin{array}{c}286,0- \\
286,4\end{array}$ & 288,00 & 289,05 & 290,00 \\
\hline $\begin{array}{c}\text { FWHM des } \\
\text { lorentziennes } \\
(\mathrm{eV})\end{array}$ & 0,2 & 0,3 & 0,3 & 0,4 & 0,5 & 0,5 \\
\hline & $\underline{\mathrm{C}-\mathrm{C}}$ & $\mathrm{C}-\mathrm{C}-\mathrm{C}=\mathrm{O}$ & $\begin{array}{c}\mathrm{C}-\mathrm{N} \\
\mathrm{O}\end{array}$ & $\mathrm{C}-\mathrm{C}=\mathrm{O}$ & $\mathrm{C}-\frac{\mathrm{C}-\mathrm{O}}{\|}$ & $\mathrm{o}-\frac{\mathrm{C}-\mathrm{O}}{\mathrm{O}}$ \\
\hline
\end{tabular}

\subsection{Préparation des échantillons}

Le Nylon 6 a été obtenu pur et sans ajout de plastifiants ou stabilisateurs auprès de Rhône Poulenc. Après avoir solubilisé le Nylon 6 dans de l'acide formique $(1 \mathrm{mg} / \mathrm{ml})$ on l'étale avec un dispositif du type barre de Conway sur une plaque en aluminium chauffée à $50^{\circ} \mathrm{C}$ pour permettre l'évaporation du solvant. Les films ainsi obtenus ont une épaisseur de $5 \mu \mathrm{m}$. La reproductibilité de la composition chimique des surfaces non traitées est vérifiée par XPS. 


\section{DÉTERMINATION DU PROFIL D'OXYGÈNe ATOMIQUe EN POST- DÉCHARGE}

La détermination du profil d'oxygène atomique et singulet le long de la post-décharge est un processus qui résulte d'une série complexe et longue de mesures expérimentales et de calculs. Ainsi que l'ont établi A. Granier et coll. [9], il faut déterminer expérimentalement la densité en oxygène atomique à l'extrémité de la décharge, base de la modélisation du milieu réactionnel en post-décharge. Les profils obtenus dans les conditions de décharge utilisées sont représentés sur la figure 3 :

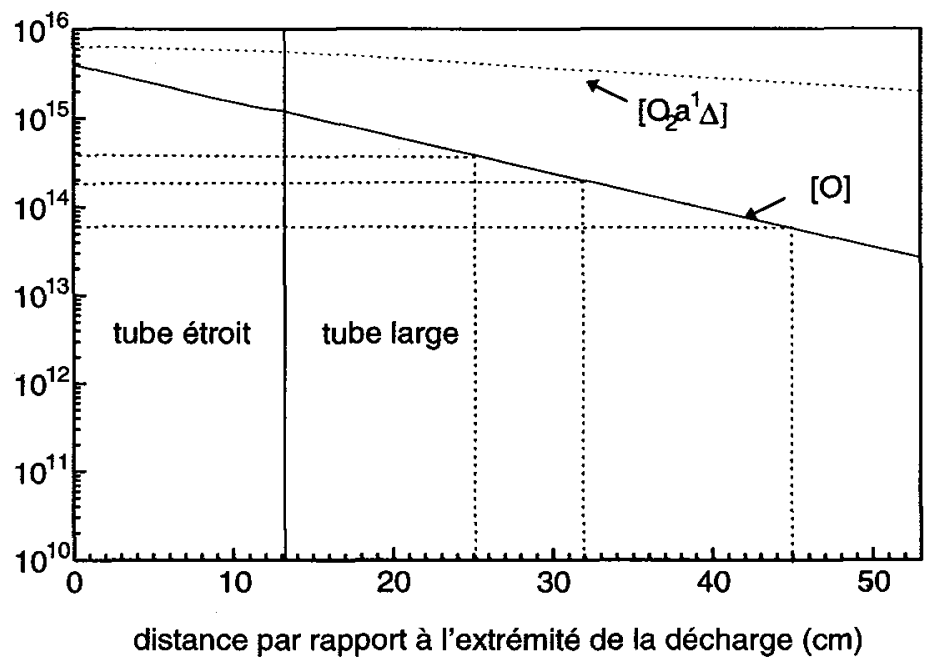

Figure 3 :Profil des densités d'oxygène atomique et moléculaire $\left(\mathrm{nbO} / \mathrm{cm}^{3}\right)$ le long de la post-décharge décrite sur la figure 2. Les points particuliers correspondent aux distances de traitements utilisées $(\$ 4.1)$

Atomic and molecular oxygen density $\left(\mathrm{nbO} / \mathrm{cm}^{3}\right)$ in the post-discharge as described in figure 2 . The particular points are related to the working distances $(\$ 4.1)$

\section{RÉSULTATS ET DISCUSSION}

\subsection{Analyse des résultats :}

Nous avons réalisé des traitements du Nylon 6 dans les conditions décrites ci-dessus. Les distances de traitement $\mathrm{X}_{2}$ sont égales à 12,19 et $32 \mathrm{~cm}$, ce qui correspond respectivement à des densités d'oxygène atomique (doses) de $4,510^{18}, 2,310^{18}$ et $6,610^{17} \mathrm{at} / \mathrm{cm}^{2} / \mathrm{s}$. On fait varier les temps de traitement de $5 \mathrm{~s}$ à $4 \mathrm{~h}$.

Les courbes présentées sur la figure 4 représentent les variations des rapports $\mathrm{O} / \mathrm{C}, \mathrm{N} / \mathrm{O}$ et N/C en fonction du temps de traitement. Il apparaît clairement qu'à temps de traitement égal les modifications sont étroitement liées aux distances $\mathrm{X}_{2}$. Les différences sont surtout marquées pour les traitements à $32 \mathrm{~cm}$ où les différents rapports atteignent assez rapidement un palier. Les évolutions des rapports à 12 et à $19 \mathrm{~cm}$ montrent que le rapport $\mathrm{O} / \mathrm{C}$ n'est pas encore 
saturé à $19 \mathrm{~cm}$ alors qu'il commence à l'être à $12 \mathrm{~cm}$ (décroissance de la valeur de $\mathrm{O} / \mathrm{C}$ après 200 min de traitement). Par ailleurs, dès les premiers instants du traitement, le rapport N/O décroît très fortement pour atteindre un palier après $30 \mathrm{~min}$ de traitement. La décroissance est accompagnée de l'augmentation des rapports $\mathrm{O} / \mathrm{C}$ et $\mathrm{N} / \mathrm{O}$ à 12 et à $19 \mathrm{~cm}$ et de la seule croissance de $\mathrm{O} / \mathrm{C}$ à $32 \mathrm{~cm}$. Elle peut s'expliquer par l'ajout d'oxygène qui diminue le taux d'azote à la surface. L'équilibre qui s'installe après $30 \mathrm{~min}$ de traitement montre soit que la quantité d'oxygène qui se lie à la surface est faible, soit qu'il y a aussi disparition de fragments azotés à la surface.

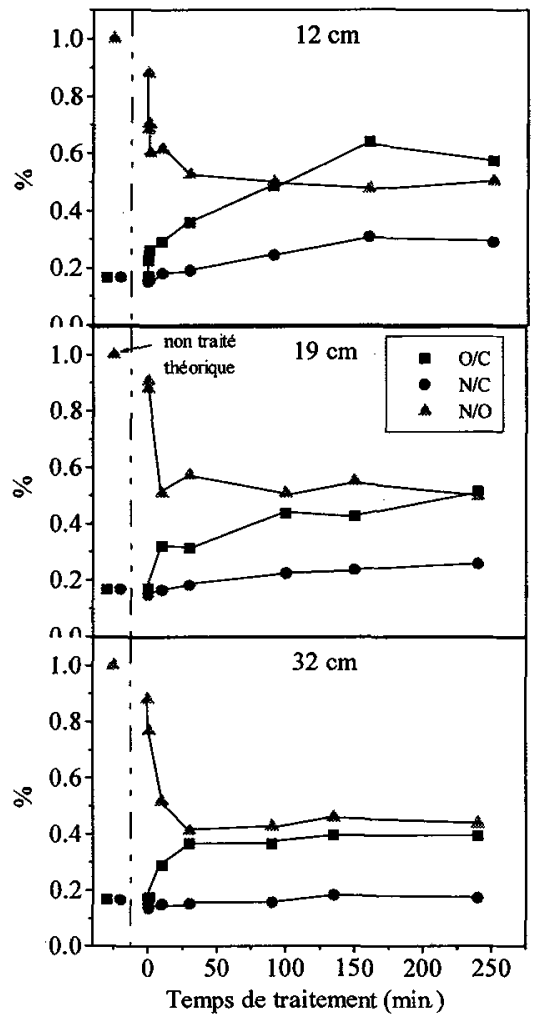

Figure 4 : Evolution des rapports $\mathrm{O} / \mathrm{C}, \mathrm{N} / \mathrm{C}$ et $\mathrm{N} / \mathrm{O}$ avec le temps de traitement (analyses in situ)

Variation of the $\mathrm{O} / \mathrm{C}, \mathrm{N} / \mathrm{C}$ et $\mathrm{N} / \mathrm{O}$ ratios with treatment time (in situ analysis)

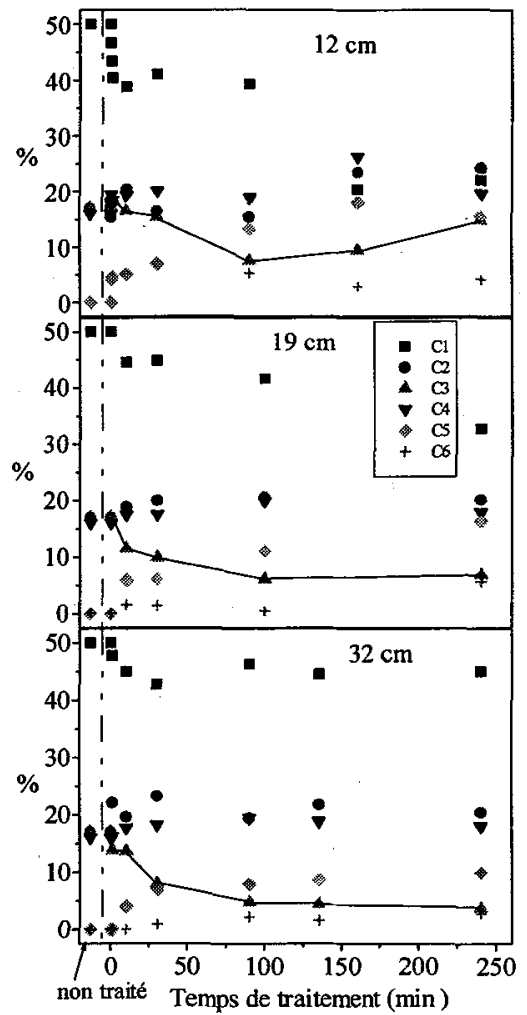

Figure 5 : Evolution des composantes des spectres $\mathrm{Cls}$ en fonction du temps de traitement (analyses in situ) Variation of the CIs components with treatment time (in situ analysis)

Les évolutions des rapports en fonction des doses d'oxygène atomique qui atteignent la surface pendant le temps de traitement soulignent le fait que le comportement d'une surface traitée à $32 \mathrm{~cm}$ diffère de celle traité à 19 ou à $12 \mathrm{~cm}$. A même dose et pour des doses très faibles, l'incorporation d'oxygène est plus forte à $32 \mathrm{~cm}$ qu'aux autres distances, mais pour des doses plus fortes, il semblerait que les distances courtes soient plus favorables à l'augmentation du rapport d'oxygène atomique. 
Les évolutions des composantes des pics C1s en fonction du temps de traitement représentées dans la figure 5 traduisent le même phénomène, à savoir qu'elles sont plus marquées à $12 \mathrm{~cm}$ qu'à $32 \mathrm{~cm}$.

L'augmentation du rapport $\mathrm{O} / \mathrm{C}$ se produit dès les premières secondes de traitement quelle que soit la distance $\mathrm{X}_{2}$, donc même pour de très faibles doses d'oxygène atomique. Elle se traduit par la création de fonctions oxygénées de type alcool, carbonyle et ester qui évoluent avec le temps de traitement vers des fonctions de type carbonate, détectées essentiellement lors de traitements très longs aux distances faibles (très grandes doses). Cependant, la stabilisation du rapport $\mathrm{O} / \mathrm{C}$ après $30 \mathrm{~min}$ de traitement ne freine pas l'évolution des structures chimiques à la surface du polymère : même si cette évolution est faible, il existe une augmentation significative de la composante C6.

\subsection{Discussion :}

Si l'on fait l'hypothèse que la quantité d'azote à la surface reste toujours la même (c'est-à-dire qu'il n'y a pas de passage des groupements amine ou amide en phase vapeur), hypothèse vérifiée par des résultats d'analyse élémentaire classique ex situ, l'augmentation du rapport $\mathrm{N} / \mathrm{C}$ ne peut s'expliquer que par une perte d'atomes de carbone à la surface. Cette perte de carbone contribue aussi à l'augmentation du rapport $\mathrm{O} / \mathrm{C}$. Ainsi, étant donné que le rapport $\mathrm{N} / \mathrm{O}$ est stable après 30 min de traitement, l'augmentation du rapport $\mathrm{O} / \mathrm{C}$ est essentiellement due à un phénomène de dégradation de la surface qui se traduit par la disparition de fragments de chaînes aliphatiques.

Ce fait est confirmé par la décomposition des spectres $\mathrm{C} 1 \mathrm{~s}$ et $\mathrm{O} 1 \mathrm{~s}$ : la composante $\mathrm{C} 3$ qui représente à la fois la liaison $\mathrm{C}-\mathrm{N}$ et la liaison $\mathrm{C}-\mathrm{O}$ diminue considérablement dans les premiers temps de traitement pour se stabiliser par la suite. Comme dans le polymère non traité, C3 représente la liaison $\mathrm{C}-\mathrm{N}$ seule, la décroissance du taux de cette composante indique bien qu'il y a coupure de cette liaison à la surface. La stabilisation qui intervient par la suite peut s'expliquer par la création de la liaison C-O. La position de la composante C3 confirme cette évolution : elle est décalée vers les plus hautes énergies de liaison au fur et à mesure du traitement. Il y a $1 \mathrm{eV}$ de décalage pour un polymère non traité (caractéristique de la liaison $\mathrm{C}$ $\mathrm{N}$ ) et jusqu'à $1,4 \mathrm{eV}$ de différence pour une surface traitée pendant un temps assez long $(t>1$ h). Par ailleurs, la composante $\mathrm{Cl}$ diminue aussi très fortement à 12 et à $19 \mathrm{~cm}$ aux temps de traitement longs. Cela peut s'expliquer par deux phénomènes : i) la création de liaisons oxydées qui diminue le taux de la composante $\mathrm{C}$, ii) la coupure et la disparition des chaînes aliphatiques qui entraîne la diminution du nombre de liaisons $\mathrm{C}-\mathrm{C}$ à la surface. En réalité, ces deux processus se réalisent de manière simultanée.

La décomposition des spectres $\mathrm{O} 1 \mathrm{~s}$ est plus délicate. La largeur anormale du pic $(2,5 \mathrm{eV}$ environ) n'est pas encore expliquée. On peut alors décomposer $\mathrm{O} 1 \mathrm{~s}$ en trois ou quatre composantes. On peut garder la décomposition en quatre composantes qui prend en compte les liaisons $\mathrm{O}-\mathrm{C}, \mathrm{O}=\mathrm{C}, \mathrm{O}-\mathrm{N}$ et $\mathrm{O}=\mathrm{N}$ puisqu'elle n'est pas incompatible avec les évolutions déjà discutées. La diminution de la composante $\mathrm{O}=\mathrm{C}$ dans les premiers temps de traitement peut s'expliquer par sa disparition mais surtout par la création de liaisons O-C. A $12 \mathrm{~cm}$, la diminution de la composante $\mathrm{C} 2$ montre l'importance des phénomènes de dégradation compatibles avec la perte de groupements carbonyle.

\subsection{Remise à l'air :}

Les résultats de la remise à l'air indiquent que suivant les traitements il y a soit une perte d'oxygène à la surface, soit un enrichissement en carbone (fig. 6). Ceci peut s'expliquer par 
l'hydrolyse des radicaux libres [10] ou par la désorption de très petites molécules qui comportent surtout des fonctions très oxydées comme le montrent la décomposition des spectres $\mathrm{C} 1 \mathrm{~s}$ (fig. 7). Ici encore, le comportement d'une surface traitée à $32 \mathrm{~cm}$ diffère de celles traitées à 12 et $19 \mathrm{~cm}$ dans le sens où les différences qui interviennent lors de la remise à l'air sur la composition de la surface sont plus marquées pour les traitements longs à 12 et à $19 \mathrm{~cm}$ et pour les traitements courts à $32 \mathrm{~cm}$. Ceci montre encore une fois l'importance des phénomènes de dégradation sur des surfaces traitées avec de fortes doses d'oxygène atomique.

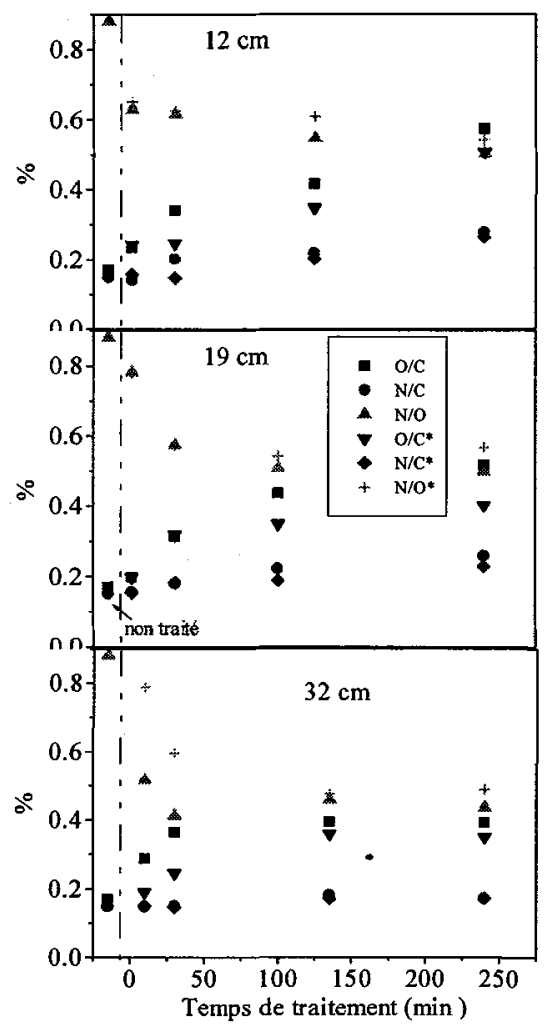

Figure 6 : Comparaison entre les rapports $\mathrm{O} / \mathrm{C}, \mathrm{N} / \mathrm{C}, \mathrm{N} / \mathrm{O}$ obtenus in situ et ex situ

Comparison of the $\mathrm{O} / \mathrm{C}, \mathrm{N} / \mathrm{C}, \mathrm{N} / \mathrm{O}$ in situ and ex situ ratios

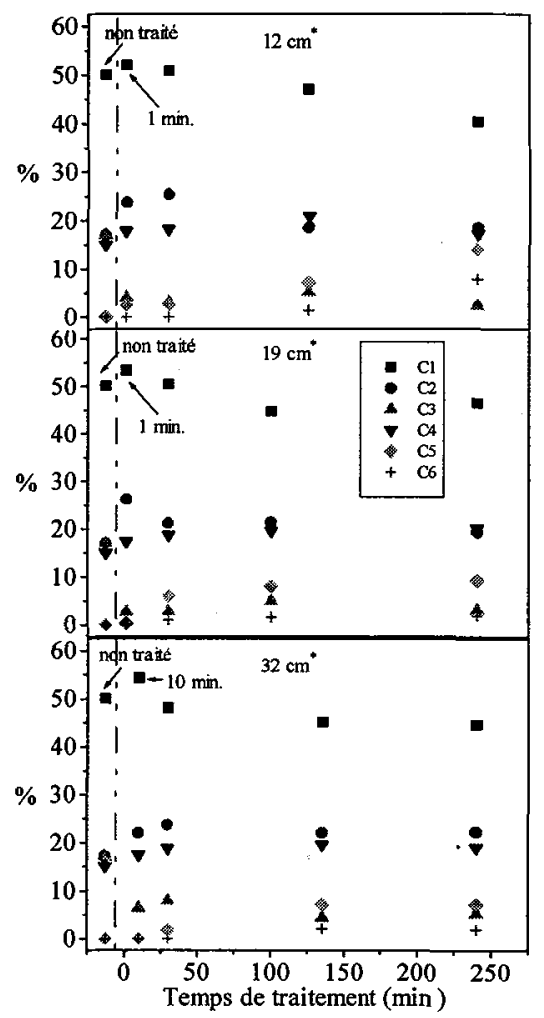

Figure 7 : Evolution des composantes des spectres Cls (analyses ex situ)

Variation of the Cls components (ex situ analysis)

\subsection{Vieillissement :}

Une étude de vieillissement sur des échantillons traités $10 \mathrm{~min}$ à $12 \mathrm{~cm}$ montre que le rapport $\mathrm{O} / \mathrm{C}$ augmente avec le temps, provoquant une chute du taux d'azote à la surface. Cette augmentation du taux d'oxygène ne se traduit pas du tout dans la décomposition des spectres $\mathrm{Cls}$ où l'on n'observe aucune variation des intensités relatives des composantes avec le temps d'exposition à l'air (les surfaces sont conservées à l'abri de la lumière). Ainsi, il ne se produit 
pas de réactions chimiques à la surface dues à l'oxygène de l'air. L'enrichissement d'oxygène à la surface serait plutôt dû à une contamination de la surface par des molécules d'eau.

\section{CONCLUSION}

Le comportement de la surface d'un film de Nylon 6 lors d'un traitement par un plasma d'oxygène en post-décharge est différent de celui des autres polymères tels que le polyéthylène ou la polycaprolactone [4] où la dégradation est un facteur limitant de la fonctionnalisation. Ici, le phénomène de dégradation qui se traduit par la coupure des chaînes aliphatiques, augmente le taux d'oxygène atomique à la surface. Il faut cependant se placer dans des conditions assez énergétiques (petites distances de traitement) pour obtenir de très fortes valeurs du rapport $\mathrm{O} / \mathrm{C}$.

Les réactions qui interviennent au moment de la remise à l'air dépendent de l'état de la surface traitée.

\section{Références}

[1] F. Poncin-Epaillard, G. Legay and J.C. Brosse J. Appl. Polym. Sci., 44 (1992) 1513.

[2] J.F. Friedrich, P. Roher, W. Saur, Th. Gross, A. Lippitz and W. Unger, Surf. Coat. Technol., 59 (1993) 371-378.

[3] A. Granier, Microwaves discharge and Applications, Plenum Publ., New York (1993) 491-501

[4] R. Prat, A. Scheuer, J. Hommet and J.P. Deville, submitted to Plasmas and Polymers.

[5] J.H. Scofield, J. Electron Spectrosc. Relat. Phenom., 8 (1976) 129.

[6] A. Jouaiti, A. Mosser, M. Romeo and S. Shindo, J. Electron Spectrosc. Relat. Phenom., 59 (1992) 327.

[7] S. Doniach and M. Sunjic, J. Phys. C, 3 (1970) 285.

[8] G. Beamson and D. Briggs, High Resolution XPS of Organic Polymers, John Wiley and Sons Ltd, Chichester, 1992).

[9] F. Normand, A. Granier, P. Leprince, J. Marec, M. K. Shi and F. Clouet, Plasma Chem. and Plasma Process.,2 (1995) 15.

[10]C. Magistrini, Thèse, Université Claude Bernard, Lyon I (1992). 